\title{
Models of Continuous-Time Linear Time-Varying Systems with Fully Adaptable System Modes
}

\author{
Miguel Ángel Gutiérrez de Anda, Arturo Sarmiento Reyes ${ }^{1}$, \\ Roman Kaszyński and Jacek Piskorowski ${ }^{2}$ \\ ${ }^{1}$ Instituto Nacional de Astrofísica, Óptica y Electrónica \\ ${ }^{2}$ Szczecin University of Technology \\ ${ }^{1}$ Mexico \\ 2Poland
}

\section{Introduction}

Linear time-varying (LTV) models have a niche of their own in the representation of systems whose behaviour may be fairly approximated as linear but whose parameters are not constant or when their behaviour shows some other properties not displayed by linear timeinvariant (LTI) models. LTV models have been applied successfully in a wide range of problems ranging from system modelling and control (see, for instance, Benton \& Smith, 2005; Kim et al., 2005; Vaishya \& Singh, 2001) or electronic circuit design (Darabi et al., 2004) to less common applications such as the modelling of soil carbon dynamics (Martin et al., 2007) or the analysis of the stability of oscillatory (bio)chemical systems (Zak et al., 2005). A common problem found in systems science and which is relevant for automation techniques and robotics is the proper identification of the parameters present in a given system. For this kind of problems, methods have been derived for the identification of LTV dynamics (see, for instance, Lortie \& Kearney, 2001). However, in particular applications where it is required to estimate in real time the parameters of a given plant with LTV behaviour and there is limited computing power for this purpose, it would be desirable to resort to another less expensive scheme.

A traditional scheme used to identify certain parameters of a given system is depicted in Figure 1. In this scheme, the output of the system under investigation $x$ is compared to the output of the adaptive filter $y$ and the difference e is used to tune the filter. Traditionally, the filter is based on an algorithm implemented on a suitable computing platform and adequate interfacing circuitry. However, nothing precludes the implementation of such a filter using analogue techniques which may lead to the synthesis of systems for analogue computing (Cowan et al., 2006). Nowadays circuit design techniques are advanced enough to synthesise in a rather convenient way all kinds of static and dynamic linear and nonlinear functions without relying in implementations comprising several operational amplifiers, resistors, capacitors and multipliers and working with low power consumption indexes and relatively high speed processing capabilities (Mulder et al., 1998). For instance, a second-order low-

Source: New Approaches in Automation and Robotics, Book edited by: Harald Aschemann, ISBN 978-3-902613-26-4, pp. 392, May 2008, I-Tech Education and Publishing, Vienna, Austria 
pass filter whose bandwidth depends linearly on the bias current $I_{0}$ is depicted in Figure 2 (Frey, 1993). It is even possible to perform with such techniques quite advanced signal processing functions like, for instance, generation of wavelet bases (Haddad et al., 2005).

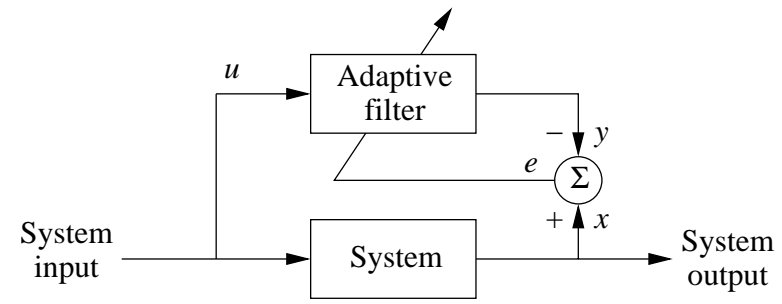

Figure 1. Parameter identification using adaptive filter

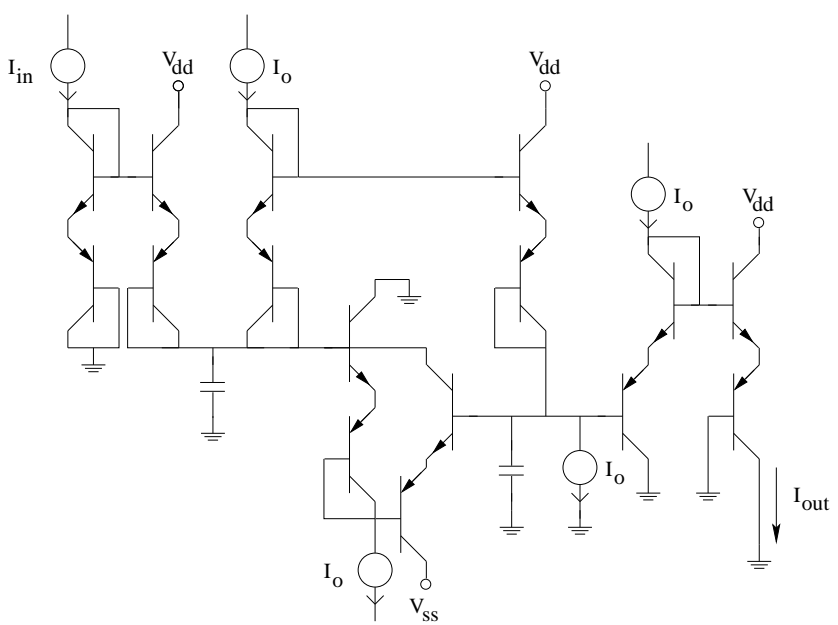

Figure 2. A second-order low-pass filter implemented with log-domain techniques (Frey, 1993).

In this chapter, a strategy to formulate continuous-time dynamical LTV systems with a prescribed dynamical behaviour will be proposed. Without any loss of generality, the strategy proposed will be first used to derive the model of a second-order LTV system with fully configurable modes. Such a model may be used to implement, for instance, an adaptive filter for system identification purposes together with an appropriate tuning scheme or may even be used for analogue computing (Cowan et al., 2006). Furthermore, the proposed model is guaranteed to have a stable behaviour provided that certain system constraints may be observed.

The rest of this chapter is organised as follows: in Section 2, a model for a second-order LTV system which may accept fast variations in its parameters without compromising its stability will be derived. In Section 3, the stability properties of the proposed model will be assessed. The properties of the new model will be compared with previous work done by the authors. The behaviour of the new model will be further validated through simulations in Section 4. Finally, some concluding remarks will be given in Section 5 . 


\section{Synthesis of continuous-time LTV models of $n$-th order}

Without any loss of generality, consider the second-order LTV differential equation

$$
x^{\prime \prime}(\mathrm{t})+\mathrm{a}_{1}(\mathrm{t}) \mathrm{x}^{\prime}(\mathrm{t})+\mathrm{a}_{0}(\mathrm{t}) \mathrm{x}(\mathrm{t})=0
$$

where $x(t)$ is a scalar function depending on the time variable $t$ with a prescribed dynamical behaviour and $a_{0}(t)$ and $a_{1}(t)$ are also scalar functions of the time variable $t$ which serve as time-varying coefficients of (1).

It is desired that the behaviour of $x(t)$ in (1) may resemble the behaviour of the scalar function $y(t)$ arising from the following LTI equation:

$$
\mathrm{y}^{\prime \prime}(\mathrm{t})+2 \xi \omega^{\prime}(\mathrm{t})+\omega^{2} \mathrm{y}(\mathrm{t})=0
$$

where $\xi$ is the damping parameter and $\omega$ is the undamped natural frequency associated to (2).

The general solution of (2) is given by

$$
y(t)=C_{1} e^{\omega\left(-\xi+\left(\xi^{2}-1\right)^{1 / 2}\right) t}+C_{2} e^{\omega\left(-\xi-\left(\xi^{2}-1\right)^{1 / 2}\right) t}
$$

where $C_{1}$ and $C_{2}$ are arbitrary constants. The general solution of $x(t)$ in (1) should resemble the behaviour of the solution of (2). However, the parameters $\xi$ and $\omega$ presented in (3) must be in general time-varying.

A possible solution is that $x(t)$ is composed by a linear combination of two modes of the form

$$
\begin{aligned}
& \mathrm{x}_{1}(\mathrm{t})=\mathrm{e}^{\int \omega(\mathrm{t})\left(-\xi(\mathrm{t})+\left(\xi^{2}(\mathrm{t})-1\right)^{1 / 2}\right) \mathrm{dt}} \\
& \mathrm{x}_{2}(\mathrm{t})=\mathrm{e}^{\int \omega(\mathrm{t})\left(-\xi(\mathrm{t})-\left(\xi^{2}(\mathrm{t})-1\right)^{1 / 2}\right) \mathrm{dt}}
\end{aligned}
$$

The proposed modes for (1) are now defined in terms of time-varying parameters. These solutions may be used to determine the coefficients $a_{1}(t)$ and $a_{0}(t)$ which are required in (1) to have the specified dynamic behaviour.

In order to obtain the time-varying coefficients of (1) from a set of known solutions, a linear algebraic system of equations has to be solved. Substitution of (4) and (5) in (1) leads to the following set of linear algebraic equations which may be solved for $\mathrm{a}_{1}(\mathrm{t})$ and $\mathrm{a}_{0}(\mathrm{t})$

$$
\left[\begin{array}{ll}
\mathrm{x}_{1}{ }^{\prime}(\mathrm{t}) & \mathrm{x}_{1}(\mathrm{t}) \\
\mathrm{x}_{2}{ }^{\prime}(\mathrm{t}) & \mathrm{x}_{2}(\mathrm{t})
\end{array}\right]\left[\begin{array}{l}
\mathrm{a}_{1}(\mathrm{t}) \\
\mathrm{a}_{0}(\mathrm{t})
\end{array}\right]=\left[\begin{array}{c}
-\mathrm{x}_{1}{ }^{\prime \prime}(\mathrm{t}) \\
-\mathrm{x}_{2}{ }^{\prime \prime}(\mathrm{t})
\end{array}\right]
$$

The solution of (6) is guaranteed to exist since $x_{1}(t)$ and $x_{2}(t)$ are linearly independent functions. After solving (6) using (4) and (5), the following expressions for $\mathrm{a}_{1}(\mathrm{t})$ and $\mathrm{a}_{0}(\mathrm{t})$ are obtained

$$
\begin{gathered}
a_{1}(t)=\xi(t)\left[2 \omega(t)-\xi^{\prime}(t)\left(\xi^{2}(t)-1\right)^{-1}\right]-\omega(t) \omega^{-1}(t) \\
a_{0}(t)=\omega(t)\left[2 \omega(t)-\xi^{\prime}(t)\left(\xi^{2}(t)-1\right)^{-1}\right]
\end{gathered}
$$


It should be noticed that other definitions for the modes of (1) could have been proposed which may resemble the dynamic behaviour of (2) when the time-varying parameters of (1) reach a constant value. Such a pair of modes would be, for instance

$$
\begin{aligned}
& \mathrm{x}_{1}(\mathrm{t})=\mathrm{e}^{\omega(\mathrm{t})\left(-\xi(\mathrm{t})+\left(\xi^{2}(\mathrm{t})-1\right)^{1 / 2}\right)} \\
& \mathrm{x}_{2}(\mathrm{t})=\mathrm{e}^{\omega(\mathrm{t})\left(-\xi(\mathrm{t})-\left(\xi^{2}(\mathrm{t})-1\right)^{1 / 2}\right)}
\end{aligned}
$$

However, such a choice would give a very complicated formulation of the functions $a_{1}(t)$ and $\mathrm{a}_{0}(\mathrm{t})$ in (1).

In general, the method used for the determination of $a_{1}(t)$ and $a_{0}(t)$ for $(1)$ may be applied to determine the time-varying coefficients $a_{i}(t), i=0,1, \ldots, n-1$ of the $n$-th order differential LTV equation of the form

$$
x^{(n)}(t)+a_{n-1}(t) x^{(n-1)}(t)+a_{n-2}(t) x^{(n-2)}(t) \ldots+a_{1}(t) x^{\prime}(t)+a_{0}(t) x(t)=0
$$

provided that $n$ linearly independent solutions $x_{i}(t), i=1,2, \ldots, n$ are known. In this case, the time-varying coefficients $a_{i}(t)$ will be the solutions of the following system of algebraic equations

$$
\left[\begin{array}{ccccc}
x_{1}(n-1)(t) & x_{1}{ }^{(n-2)}(t) & \ldots & x_{1}{ }^{\prime}(t) & x_{1}(t) \\
x_{2}(n-1)(t) & x_{2}{ }^{(n-2)}(t) & \ldots & x_{2}{ }^{\prime}(t) & x_{2}(t) \\
\cdots & \cdots & \ldots & \cdots & \cdots \\
x_{n-1}(n-1)(t) & x_{n-1}(n-2)(t) & \ldots & x_{n-1}{ }^{\prime}(t) & x_{n-1}(t) \\
x_{n}{ }^{(n-1)}(t) & x_{n}{ }^{(n-2)}(t) & \ldots & x_{n}{ }^{\prime}(t) & x_{n}(t)
\end{array}\right] \quad\left[\begin{array}{c}
a_{n-1}(t) \\
a_{n-2}(t) \\
\cdots \\
a_{1}(t) \\
a_{0}(t)
\end{array}\right]=\left[\begin{array}{c}
-x_{1}(n)(t) \\
-x_{2}(n)(t) \\
\cdots \\
-x_{n-1}(n)(t) \\
-x_{n}(n)(t)
\end{array}\right]
$$

In this particular case, the existence of the solutions of this system of equations is guaranteed to exist since the solutions $x_{i}(t)$ are linearly independent and therefore their Wronksian will be different from zero for all $t$. In the formulation of a model with set of fully configurable modes, and depending on the expected dynamics of the system to be modelled, the modes given in expressions (4) and (5) are excellent candidates to represent a couple of complex conjugate poles whose exact locations are not known. On the other hand, the function $x(t)=e^{-f(t) d t}$ with $\sigma(t)>0$ may be used to represent a real pole located in the left plane. As it will be seen soon, the proposed functions represent the best alternative to build a system based on equation (11) with complex dynamical behaviour.

In previous work (Kaszyński, 2003; Jaskula, \& Kaszyński, 2004; Piskorowski, 2006), the following equation was proposed for defining a new class of low-pass time-varying filters

$$
x^{\prime \prime}(t)+2 \xi(t) \omega(t) x^{\prime}(t)+\omega^{2}(t) x(t)=\omega^{2}(t) u(t)
$$

where $u(t)$ is the signal to be filtered, $x(t)$ represents the output signal and $\xi(t)$ and $\omega(t)$ represent time-varying parameters. If the coefficients of (12) are compared to the coefficients defined in (7) and (8) for (1), it can be concluded that the system defined in (1) will approximate the behaviour of (12) when the maximum magnitude of the logarithmic derivative of function $\omega(t)$ and the maximum magnitude of function $\xi^{\prime}(t) /\left(\xi^{2}(t)-1\right)$ are small compared to $2 \xi(t) \omega(t)$ and $\omega(t)$, respectively. In other words, $\xi(t)$ and $\omega(t)$ have to vary slowly. 


\section{Stability properties of the proposed model}

The stability properties of $(1)$ when $\mathrm{a}_{1}(\mathrm{t})$ and $\mathrm{a}_{0}(\mathrm{t})$ are defined according to (7) and (8) are determined solely by the modes used in the formulation of these coefficients. In the general case (i.e. when time-varying parameters $\xi(t)$ and $\omega(t)$ vary arbitrarily), a suitable measure of the stability of the modes given in (4) and (5) can be obtained if their Lyapunov exponents are computed. The Lyapunov exponents $L_{1}$ and $L_{2}$ associated to modes $x_{1}(t)$ and $x_{2}(t)$ in $(4)$ and (5) are given by

$$
\begin{aligned}
& \mathrm{L}_{1}=\lim _{\mathrm{t} \rightarrow \infty} \sup \mathrm{t}^{-1} \ln \left|\mathrm{x}_{1}(\mathrm{t})\right|=\lim \operatorname{Ret}^{-1} \int \omega(\mathrm{t})\left[-\xi(\mathrm{t})+\left(\xi^{2}(\mathrm{t})-1\right)^{1 / 2}\right] \mathrm{dt} \\
& \mathrm{L}_{2}=\lim _{\mathrm{t} \rightarrow \infty} \sup \mathrm{t}^{-1} \ln \left|\mathrm{x}_{2}(\mathrm{t})\right|=\lim \operatorname{Ret}^{-1} \int \omega(\mathrm{t})\left[-\xi(\mathrm{t})-\left(\xi^{2}(\mathrm{t})-1\right)^{1 / 2}\right] \mathrm{dt}
\end{aligned}
$$

In order to assure the stability of $(1), \mathrm{L}_{1}<0$ and $\mathrm{L}_{2}<0$. Therefore, it suffices that the real part of those time averages remains negative. This means that function $\xi(t)$ may take arbitrary negative values and the stability of the system will be still guaranteed, provided that the average value of $\omega(\mathrm{t})\left[-\xi(\mathrm{t}) \pm\left(\xi^{2}(\mathrm{t})-1\right)^{1 / 2}\right]$ is negative over the whole time interval in which (1) is operating. This gives added flexibility when implementing a control for modulating $\xi(\mathrm{t})$ and $\omega(\mathrm{t})$ according to a predefined rule.

An important issue behind the proposed model in (1) is the possibility that it might be bounded-input bounded-output (BIBO) stable when the right hand of (1) contains a nonzero term. In order to guarantee this type of stability, it suffices that the homogeneous response of (1) is exponentially asymptotically stable and that the coefficients $a_{0}(t)$ and $a_{1}(t)$ are bounded for all $t$ (Anderson \& Moore, 1969). In this case, both conditions are satisfied and therefore the proposed model has BIBO stability. The proposed modes in expressions (4) and (5) have exponential asymptotic stability provided that $\omega(t)$ and $\xi(t)$ remain positive for all $t$. In order to guarantee the boundedness of the coefficients of $(1), \omega(t)$ hast to be different from zero for all $t$, whereas $\xi(t)$ has to be different from 1 for all $t$.

In the particular case of the system governed by equation (11), the considerations given in (Anderson \& Moore, 1969) to guarantee its BIBO stability when the right hand of (11) is nonzero are also applicable. It should be noticed that if the dynamics of the system should include a real pole in the left plane whose location is not known, function $x(t)=e^{-f \sigma(t) d t}$ has also exponential asymptotic stability provided that $\sigma(t)>0$ for all $t$. Moreover, the average value of $\sigma(t)$ will define the Lyapunov exponent associated to that solution.

The results obtained so far should be compared against previous work. In (Kaszyński, 2003), the following conditions for assuring the stability of (12) were given

$$
\begin{gathered}
\omega(\mathrm{t})>0 \\
\xi(\mathrm{t})>0 \\
\left|\omega^{\prime}(\mathrm{t})\right|<\left|2 \xi(\mathrm{t}) \omega^{2}(\mathrm{t})\right|
\end{gathered}
$$

Such conditions were obtained using the second Lyapunov method. Conditions (15) and (16) are sufficient in (1) to ensure stability for all t. With these conditions, it is guaranteed that the arguments of the integrals in (13) and (14) are always negative, and therefore the 
Lyapunov exponents computed in this way will be also negative. Condition (17) imposes an additional constraint on (12): in order to ensure stability, $\omega(t)$ may not be varied above the limit imposed by the product $2 \xi(\mathrm{t}) \omega^{2}(\mathrm{t})$, thus limiting the tunability range of this parameter. System (1) with coefficients $a_{1}(t)$ and $a_{0}(t)$ as defined in (7) and (8) does not show the shortcoming described above.

To further demonstrate that the dynamics of (1) and (12) are different, the sum of the Lyapunov exponents will be computed. According to (Nemytskii \& Stepanov, 1989), the sum of the Lyapunov exponents $\mathrm{v}$ of a regular $\mathrm{n}$-th order LTV system of the form (11) is given by

$$
v=-\lim _{\mathrm{t} \rightarrow \infty} \sup \mathrm{t}^{-1} \int_{0}^{\mathrm{t}} \mathrm{a}_{\mathrm{n}-1}(\tau) \mathrm{d} \tau
$$

If the coefficient associated to the $x^{\prime}(t)$ term of (12) is compared to (7), it will be verified that the only way to make the sum of Lyapunov exponents equal for both systems is forcing the product $2 \xi(t) \omega(t)$ to be the same in both systems and cancelling the time variations of $\xi(t)$ and $\omega(t)$. This means that if the sum of Lyapunov exponents for (1) and (12) is meant to be the same, both systems must be LTI and have the same set of eigenvalues.

\section{Performance evaluation}

In order to verify the stability properties of the proposed model, its response will be tested for a predefined test signal (a step function). Therefore, $x(t)$ will be estimated numerically from the following equation

$$
x^{\prime \prime}(t)+a_{1}(t) x^{\prime}(t)+a_{0}(t) x(t)=a_{0}(t) u(t)
$$

where coefficients $\mathrm{a}_{1}(\mathrm{t})$ and $\mathrm{a}_{0}(\mathrm{t})$ are defined as in (7) and (8) and $\mathrm{u}(\mathrm{t})$ is the step function. The obtained response will be compared to the response obtained from a reference LTI system and from the LTV filter based on equation (12) subject to the same parameter variation. It will be assumed that the LTI reference filter is a second-order lowpass Butterworth filter with poles located on $-5 \pm 5 \mathrm{j}$. Furthermore, it will be assumed that $\omega(t)$ and $\xi(\mathrm{t})$ are given by the following expressions

$$
\begin{gathered}
\omega(t)=\left[50+50 \mathrm{e}^{-40 t}+100 \mathrm{e}^{-20 t}\right]^{1 / 2} \\
\xi(t)=\left(10+10 \mathrm{e}^{-20 t}\right) / 2 \omega(\mathrm{t})
\end{gathered}
$$

Although it has been already demonstrated a long time ago that the eigenvalues obtained from an arbitrary LTV system by means of the well-known characteristic equation for LTI systems do not convey any valid information regarding the stability properties of the system or its solution (Vinogradov, 1952), with the definition given for $\omega(t)$ and $\xi(t)$ in (20) and (21) the instantaneous location of the roots of the characteristic equation associated to (12) is modulated exponentially in time from $-10 \pm 10 \mathrm{j}$ at $\mathrm{t}=0$ to $-5 \pm 5 \mathrm{j}$ within a relatively short time interval. With a time constant of 0.05 seconds for the modulation factor, the roots associated to the characteristic equation of (12) should be very close to the poles of the reference LTI system after 0.2 seconds.

The simulated responses obtained in Mathematica for the LTI reference system, the filter described based on (12) and the proposed LTV model are depicted in Figure 3. From the 
simulation results it can be seen that the responses of both LTV systems become identical to the response of the LTI reference system as t increases. Although the response generated by (12) rises faster compared to the response generated by (19), the proposed model produces a smaller overshoot.

In Figure 4, the response to the step function of the systems defined by (19) and (12) is depicted. In this particular case, it was assumed that $\omega(\mathrm{t})$ and $\xi(\mathrm{t})$ were varied according to the following relations

$$
\begin{gathered}
\omega(t)=\left[50+90 \sin (10 \Pi t)+40.5 \sin ^{2}(10 \Pi t)\right]^{1 / 2} \\
\xi(t)=[10+9 \sin (10 \Pi t)] / 2 \omega(t)
\end{gathered}
$$

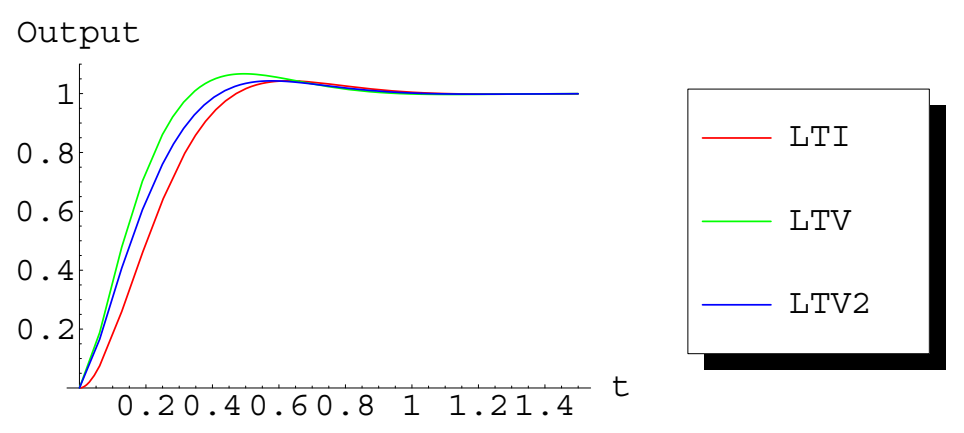

Figure 3. Responses of (12) and (19) to a step response with an exponential variation of $\omega(t)$ and $\xi(t)$. In this figure, the acronym LTV stands for the response of (12) whereas the acronymLTV2 stands for the response of (19)

With the chosen variation parameters for $\omega(t)$ and $\xi(t)$, the roots of the characteristic equation associated to (12) will vary periodically according to the following relation

$$
\mathrm{p}_{1,2}=-5 \pm 5 j+(-4.5 \pm 4.5 \mathrm{j}) \cos (10 \pi \mathrm{t})
$$

where $p_{1}$ and $p_{2}$ stand for the roots of the characteristic equation of (12). With this variation scheme, the condition given in (17) for (12) is not valid anymore. However, in the case of (19), the stability measures given in (13) and (14) for equation (1) $\left(\mathrm{L}_{1}=\mathrm{L}_{2}=-5\right.$ ), the exponential asymptotic nature of its homogeneous response and the boundedness of its coefficients guarantee that the output of (19) will be bounded (Anderson \& Moore, 1969). Given that (12) is expected to show an unstable behaviour, backward differentiation formulae were chosen to solve numerically equations (19) and (12). From the results of the simulation it can be seen that both LTV systems reach in a finite time their steady-state behaviour. However, the system described by (12) displays a somewhat larger overshoot before it reaches steady-state behaviour.

It is noteworthy to consider the role of the scaling coefficient of the input function $u(t)$ in equations (12) and (19). According to condition (17), the output of equation (12) should be unstable if the coefficients given in expressions (23) and (24) are considered. However, the simulation results of Figure 4 show that the output of the filter described by equation (12) is 
stable. If the scaling coefficient of the input function $\mathrm{u}(\mathrm{t})$ present in equation (12) is removed and the following equation is instead considered

$$
x^{\prime \prime}(t)+2 \xi(t) \omega(t) x '(t)+\omega^{2}(t) x(t)=u(t)
$$

it is possible to demonstrate that the stability condition given in (17) is indeed broken.
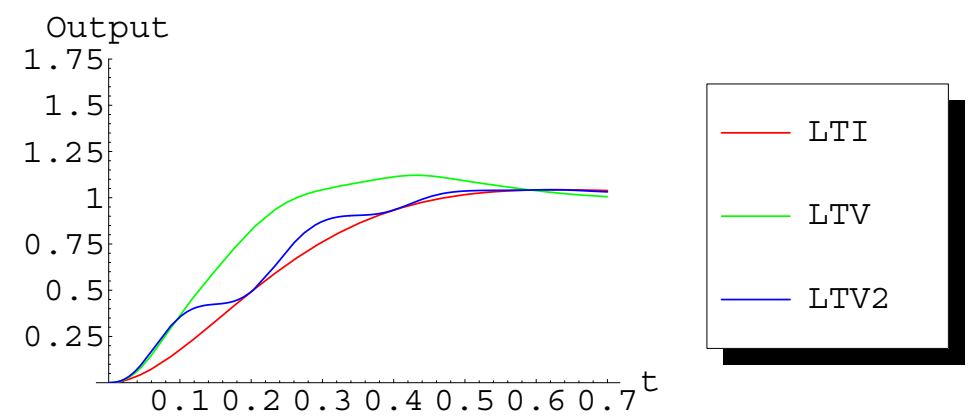

Figure 4. Responses of (12) and (19) to a step response with a periodic variation of $\omega(t)$ and $\xi(\mathrm{t})$.

\section{Output}

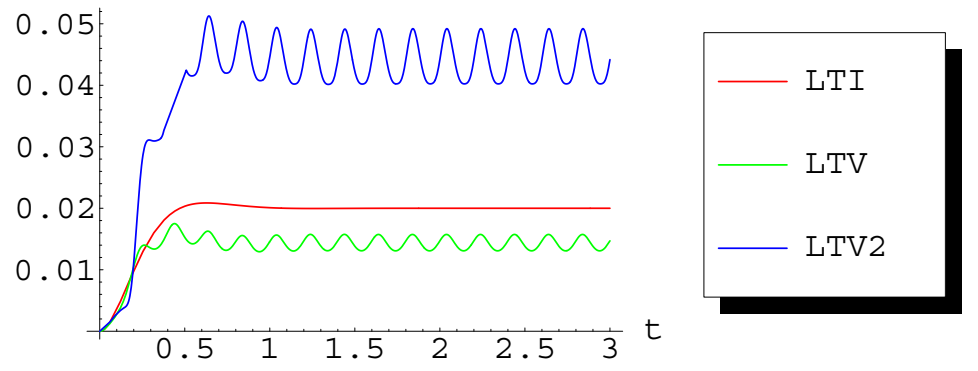

Figure 5. Responses of (25) and (26) to a step response with a periodic variation of $\omega(t)$ and $\xi(\mathrm{t})$

In Figure 5, the response of equation (25) with periodic coefficients $\omega(t)$ and $\xi(t)$ as defined in (23) and (24) to a unit step function is depicted. This response is compared to the response of the LTV differential equation

$$
x^{\prime \prime}(t)+a_{1}(t) x '(t)+a_{0}(t) x(t)=u(t)
$$

and to the response of the lowpass filter described by the following LTI differential equation

$$
\mathrm{x}^{\prime \prime}(\mathrm{t})+50 \mathrm{x} \cdot(\mathrm{t})+10 \mathrm{x}(\mathrm{t})=\mathrm{u}(\mathrm{t})
$$

In expression (26), coefficients $\mathrm{a}_{0}(\mathrm{t})$ and $\mathrm{a}_{1}(\mathrm{t})$ are defined as given in (8) and (7). From Figure 5 it may be noticed that the responses of equations (25) and (26) are oscillatory and do not track the input function $\mathrm{u}(\mathrm{t})$. In this case, function $\mathrm{u}(\mathrm{t})$ is assumed to be a step function. 


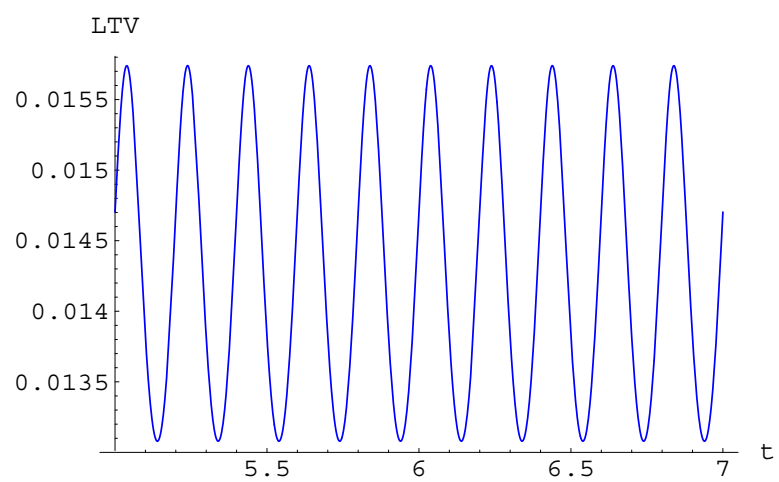

Figure 6. Time series considered for the output of (25)

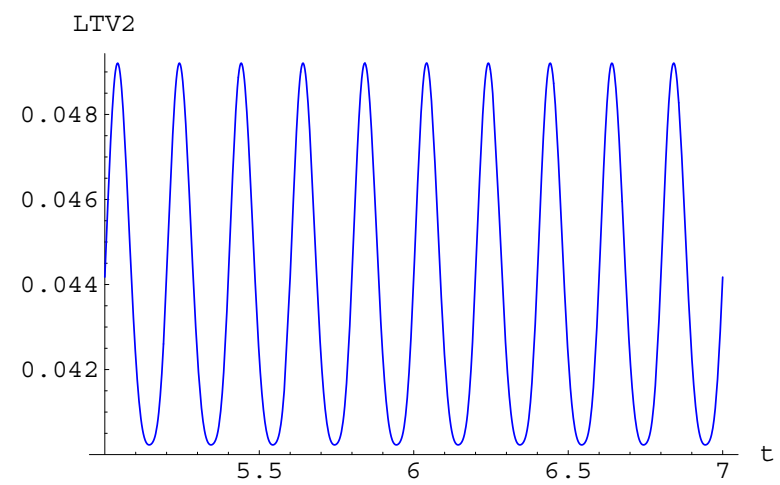

Figure 7. Time series considered for the output of (26)

A detailed analysis of a portion of the time series obtained from (25) and (26) gives more information on the results obtained through simulation with Mathematica. The time series considered for analysis appear in Figures 6 and 7. In a first attempt, Lyapunov exponents may be determined to establish the nature of the behaviour displayed by the systems represented by equations (25) and (26). However, given the periodic character of their time series, other methods should be used to assess the stability of (25) and (26) (Sprott, 2003).

In order to determine whether the stability condition given in (17) is broken or not for equation (25), the power spectra of the time series considered should be obtained. According to (Sigeti, 1995), the power spectrum of a given signal with positive Lyapunov exponents has an exponential high-frequency falloff relationship. Such characteristic in the frequency domain is due to the fact that the function which defines the signal under consideration has singularities in the complex plane when the time variable $t$ is seen as a complex variable and not as a real one (Sigeti, 1995). When the Fourier transform is computed for such a signal, the singularities must be avoided in the complex plane through an adequate integration path and in this way exponential terms appear on its associated Fourier transform (Sigeti, 1995). In the presence of noise, the exponential frequency falloff relationship will be noticeable up to a given frequency and afterwards it will decay as a power of $\mathrm{f}^{-\mathrm{n}}$ where $\mathrm{f}$ is the frequency and $n$ a natural number (Lipton \& Dabke, 1996). These phenomena are also observable in chaotic systems as well, independently of the appearance of attractors or not 
in their dynamic behaviour (van Wyk \&. Steeb, 1997). When there are no singularities in the complex variable $t$ present in a given signal and in the absence of noise, its power spectrum will decay at high frequencies as a power of $\mathrm{f}^{-\mathrm{n}}$ as well (Sigeti \& Horsthemke, 1987).

\begin{tabular}{|c|c|}
\hline Frequency $(\mathrm{Hz})$ & Magnitude \\
\hline 0 & $2.86448 \times 10^{-2}$ \\
\hline 5 & $1.32849 \times 10^{-3}$ \\
\hline 10 & $8.75641 \times 10^{-5}$ \\
\hline 15 & $2.82938 \times 10^{-6}$ \\
\hline 20 & $4.9275 \times 10^{-8}$ \\
\hline 25 & $1.02782 \times 10^{-9}$ \\
\hline
\end{tabular}

Table 1. Discrete power spectrum for the output of equation (25)

\begin{tabular}{|c|c|}
\hline Frequency $(\mathrm{Hz})$ & Magnitude \\
\hline 0 & $8.78076 \times 10^{-2}$ \\
\hline 5 & $4.50563 \times 10^{-3}$ \\
\hline 10 & $8.13031 \times 10^{-4}$ \\
\hline 15 & $5.94339 \times 10^{-5}$ \\
\hline 20 & $7.58989 \times 10^{-6}$ \\
\hline 25 & $2.38456 \times 10^{-6}$ \\
\hline 30 & $8.0822 \times 10^{-7}$ \\
\hline 35 & $3.04263 \times 10^{-7}$ \\
\hline 40 & $1.23233 \times 10^{-7}$ \\
\hline 45 & $5.27326 \times 10^{-8}$ \\
\hline 50 & $2.35502 \times 10^{-8}$ \\
\hline 55 & $1.08831 \times 10^{-8}$ \\
\hline 60 & $5.1718 \times 10^{-9}$ \\
\hline
\end{tabular}

Table 2. Discrete power spectrum for the output of equation (26)

Given that the numerical solutions obtained for equations (25) and (26) are periodic, their power spectra turn out to be discrete. In Tables 1 and 2 the magnitude of the harmonic components of the responses computed via Mathematica has been tabulated. The data given in Table 1 was used to obtain the best fit in Mathematica using routine NonlinearFit[ ] for expressions

and

$$
\mathrm{y}=\mathrm{A}_{1} \mathrm{e}^{\mathrm{B} 1 \mathrm{f}}
$$

$$
\mathrm{y}=\mathrm{A}_{2} \mathrm{f}^{\mathrm{B}} 2
$$

where $A_{1}, A_{2}, B_{1}$ and $B_{2}$ are fitting parameters. If the data given in Table 1 is considered from $\mathrm{f}=15 \mathrm{~Hz}$ for the fitting process, the constants $A_{1}$ and $B_{1}$ which fit best expression (28) are equal to 0.535465 and -0.810056 respectively. With the same data, the constants obtained for the best fit of expression (29) are $A_{2}=175010$ and $B_{2}=-9.17964$. In Figure 8 expressions (28) and (29) are plotted together with the original data and it can be seen that the exponential curve matches better the obtained data from equation (25) at high frequencies. The same procedure was carried out with the data presented in Table 2. The coefficients obtained for expression (28) were $A_{1}=0.0244961$ and $B_{1}=-0.401458$ whereas for expression (29) the coefficients were $A_{2}=10292.2$ and $B_{2}=-7.00509$. From Figure 9 it can be seen that 


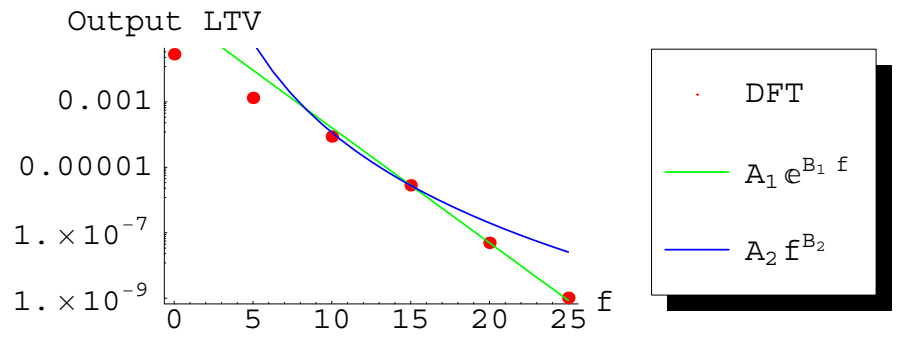

Figure 8. Power spectrum obtained for the time series of (25)
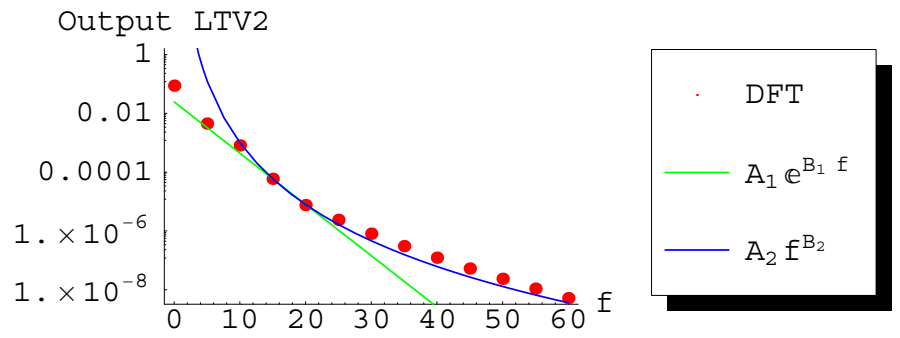

Figure 9. Power spectrum obtained for the time series of (26)

expression (29) gives the best fit for the data obtained from equation (26) at frequencies greater than $15 \mathrm{~Hz}$. Given that condition (17) is broken, it can be thus safely concluded that the response obtained from equation (26) is unstable when $\omega(t)$ and $\xi(t)$ are defined as given in expressions (23) and (24). In the case of equation (26), under the same conditions, it turns out that its response is bounded. The response of equation (26) is bounded for a bounded input because the conditions given in (Anderson \& Moore, 1969) for BIBO stability are enforced.

\section{Conclusions}

In this chapter, a strategy for the formulation of a LTV scalar dynamical system with predefined dynamic behaviour was presented. Moreover, a model of a second-order LTV system whose dynamic response is fully adaptable was presented. It was demonstrated that the proposed model has a exponentially asymptotically stable behaviour provided that a set of stability constraints are observed. Moreover, it was demonstrated that the obtained system is BIBO stable as well. Finally, it was shown via simulations that the response of the proposed model reaches with a smaller overshoot its steady-state response compared to the response of a LTV lowpass filter proposed previously.

\section{References}

Anderson, B. \& Moore, J. (1969). “New results in linear system stability," SIAM Journal of Control, vol. 7, no. 3, pp. 398-414. 
Benton, R.E \& Smith, D. (2005). “A static-output-feedback design procedure for robust emergency lateral control of a highway vehicle," IEEE Transactions on Control Systems Technology, vol. 13, no. 4, pp. 618-623.

Cowan G.E.R.; Melville, R.C.; \& Tsividis, Y.P. (2006) “A VLSI analog computer/digital computer accelerator," IEEE Journal of Solid-State Circuits, vol. 41, no. 1, pp. 42-53.

Darabi, H.; Ibrahim, B. Rofougaran A. (2004). "An analog GFSK modulator in 0.35- $\mu \mathrm{m}$ CMOS," IEEE Journal of Solid-State Circuits, vol. 39, no. 12, pp. 2292-2296.

Frey, D.R. (1993). "Log-domain filtering: an approach to current-mode filtering," IEE Proceedings G: Circuits, Devices and Systems, vol. 43, no. 6, pp. 403-416.

Haddad, S.A.P.; Bagga, S. \& Serdijn, W.A. (2005). "Log-domain wavelet bases," IEEE Transactions on Circuits and Systems I-Regular Papers, vol. 52, no. 10, pp. 2023-2032.

Jaskula, M. \& Kaszyński, R. (2004). "Using the parametric time-varying analog filter to average evoked potential signals," IEEE Transactions on Instrumentation and Measurement, vol. 53, no. 3, pp. 709-715.

Kaszyński, R (2003). "Properties of analog systems with varying parameters [averaging/low-pass filters]," in Proceedings of the 2003 International Symposium on Circuits and Systems 2003, vol. 1, pp. 509-512.

Kim, W.; Lee, D.-J.; \& Chung, J. (2005). “Three-dimensional modelling and dynamic analysis of an automatic ball balancer in an optical disk drive," Journal of Sound and Vibration, vol. 285, pp. 547-569.

Lipton, J.M. \& Dabke, K.P. (1996)."Reconstructing the state space of continuous time chaotic systems using power spectra," Physics Letters A, vol. 210, pp. 290-300.

Lortie, J.M. \& Kearney, R.E. (2001). “Identification of physiological systems: estimation of linear time-varying dynamics with non-white inputs and noisy outputs," Medical and Biological Engineering and Computing, vol. 39, pp. 381-390, 2001.

Martin, M.P.; Cordier, S.C.; Balesdent, J. \& Arrouays D. (2007). "Periodic solutions for soil carbon dynamics equilibriums with time-varying forcing variables," Ecological modelling, vol. 204, no. 3-4, pp. 523-530.

Mulder, J.; Serdijn, W.A.; van der Woerd, A. \& van Roermund, A.H.M. (1998). Dynamic translinear and log-domain circuits: analysis and synthesis, Kluwer Academic Publishers, Dordrecht, The Netherlands.

Nemytskii, V.V. \& Stepanov, V.V. (1989). Qualitative theory of differential equations, Dover, NewYork, 1989.

Piskorowski, J. (2006). "Phase-compensated time-varying Butterworth filters," Analog Integrated Circuits and Signal Processing, vol. 47, no. 2, pp. 233-241.

Sigeti, D.E. (1995). "Exponential decay of power spectra at high frequency and positive Lyapunov exponents," Physica D, vol. 82, pp. 136-153.

Sigeti, D. \& Horsthemke, W. (1987). "High-frequency power spectra for systems subject to noise," Physical Review A, vol. 35, no. 5, pp. 2276-2282.

Sprott, J.C. (2003). Chaos and time-series analysis, Oxford University Press, Oxford.

Vaishya, M.\& Singh R. (2001). "Sliding friction-induced nonlinearity and parametric effects in gear dynamics," Journal of Sound and Vibration, vol. 248, pp. 671-694.

van Wyk, M.A. \&. Steeb, W.-H. (1997). Chaos in Electronics, Kluwer, Dordrecht, the Netherlands.

Vinogradov, R.E. (1952), “On a criterion for instability in the sense of A.M. Lyapunov for solutions of linear systems of differential equations," Dokl. Akad. Nauk SSSR, vol. 84 , no. 2.

Zak, D.E.; Stelling, J. \& Doyle III, F.J. (2005). “Sensitivity analysis of oscillatory (bio)chemical systems," Computers and Chemical Engineering, vol. 29, pp. 663-673. 


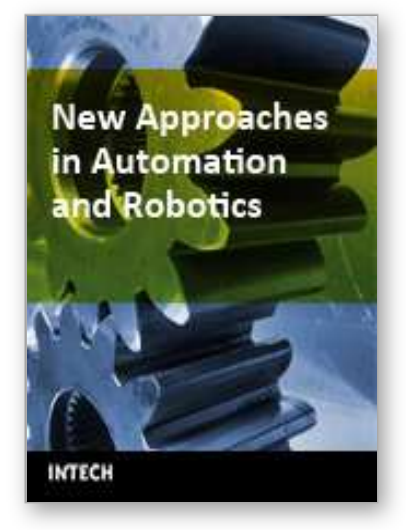

\author{
New Approaches in Automation and Robotics \\ Edited by Harald Aschemann
}

ISBN 978-3-902613-26-4

Hard cover, 392 pages

Publisher I-Tech Education and Publishing

Published online 01, May, 2008

Published in print edition May, 2008

The book New Approaches in Automation and Robotics offers in 22 chapters a collection of recent developments in automation, robotics as well as control theory. It is dedicated to researchers in science and industry, students, and practicing engineers, who wish to update and enhance their knowledge on modern methods and innovative applications. The authors and editor of this book wish to motivate people, especially under-graduate students, to get involved with the interesting field of robotics and mechatronics. We hope that the ideas and concepts presented in this book are useful for your own work and could contribute to problem solving in similar applications as well. It is clear, however, that the wide area of automation and robotics can only be highlighted at several spots but not completely covered by a single book.

\title{
How to reference
}

In order to correctly reference this scholarly work, feel free to copy and paste the following:

Miguel Angel Gutierrez de Anda, Arturo Sarmiento Reyes, Roman Kaszynski and Jacek Piskorowski (2008). Models of Continuous-Time Linear Time-Varying Systems with Fully Adaptable System Modes, New Approaches in Automation and Robotics, Harald Aschemann (Ed.), ISBN: 978-3-902613-26-4, InTech, Available from:

http://www.intechopen.com/books/new_approaches_in_automation_and_robotics/models_of_continuoustime_linear_time-varying_systems_with_fully_adaptable_system_modes

\section{INTECH}

open science | open minds

\author{
InTech Europe \\ University Campus STeP Ri \\ Slavka Krautzeka 83/A \\ 51000 Rijeka, Croatia \\ Phone: +385 (51) 770447 \\ Fax: +385 (51) 686166 \\ www.intechopen.com
}

\author{
InTech China \\ Unit 405, Office Block, Hotel Equatorial Shanghai \\ No.65, Yan An Road (West), Shanghai, 200040, China \\ 中国上海市延安西路65号上海国际贵都大饭店办公楼 405 单元 \\ Phone: +86-21-62489820 \\ Fax: $+86-21-62489821$
}


(C) 2008 The Author(s). Licensee IntechOpen. This chapter is distributed under the terms of the Creative Commons Attribution-NonCommercialShareAlike-3.0 License, which permits use, distribution and reproduction for non-commercial purposes, provided the original is properly cited and derivative works building on this content are distributed under the same license. 